\title{
An exploration of mammographers' attitudes towards the use of Social Media for providing breast screening information to clients
}

\section{Introduction}

The authors of this study have created an online hub (www.wommen.org.uk) which provides information about breast screening. The aim of the hub is to address the reported limitations of the information currently sent to women in the United Kingdom (UK) when invited for breast screening [1,2]. In the design phase of the hub, we engaged a client and practitioner User Design Group (UDG) to identify the key features that would be desirable in the hub [3]. One such feature was the need to engage with other clients in order to access experiential information, and with practitioners to access 'professional' information. Social Media (SoMe) has therefore been incorporated and is an important element of the hub because it allows communication between clients and practitioners using forums and also via links to associated Facebook and Twitter accounts.

SoMe is any web-based application that allows users to create and share content. Ofcom, the UK communications regulator, produces annual statistics of adult SoMe use [4]. Their 2016 report highlighted that the majority of the UK adult population who we are aiming to target with WoMMeN (females in the 45-54 age group; Ofcom's age categories) is likely to be using SoME and that this trend is rising. Seventy eight percent of all adult females had a SoMe profile in 2015 compared to $56 \%$ in 2010 . In terms of the age bracket we are concerned with, $74 \%$ of $45-54$ year olds had a social media profile in 2015 compared to $32 \%$ in 2010. This suggests using SoMe to communicate with breast screening clients could be an appropriate medium.

Furthermore, the use of online methods for communicating with patients is also advocated in the 2012 UK NHS communication strategy [5]. However, as the literature review will show, there appears to be reluctance for health professionals to talk to patients and clients in an online space. This needs to be examined further because without practitioners the online hub will not include the communication features identified as desirable by the UDG.

\section{Literature Review}

To date there have been no studies published that have explored the attitudes of radiographers with regard to using SoMe as a professional tool [6]. However, a number of systematic reviews have considered how SoMe is being used across a wider range of health-related disciplines. 
In 2013, Moorhead et al conducted a systematic review looking into the uses, benefits and limitations of SoMe for Health Communication [7]. They reviewed 98 studies and highlighted the value of SoMe in health for connecting and networking people: practitioners with patients; patients with patients and practitioners with practitioners. The benefits identified were i) enabling peer and emotional support and ii) sharing information that is tailored to the recipient, not just the patient but their relatives and carers. However, there was a clear message that practitioners have anxieties about SoMe as a means of health communication. These concerned quality of information and issues related to privacy and patient confidentiality.

In 2014, Grajales et al [8] conducted a narrative review to explore how SoMe is being used by health professionals. The study included 76 articles, 44 websites and 11 policy documents. Like Moorhead et al, this study showed that whilst there were many cases of innovative practices using a range of SoMe platforms, there was still widespread confusion and fear amongst health practitioners. These related primarily to professional issues such as confidentiality, patient privacy and breaching professional and ethical codes of conduct. There was also variation seen in the way professional bodies and employers supported the use of SoMe for professional communication, with some advocating and others condemning its use.

Lawson and Cowling's 2014 systematic review [6] also looked at SoMe as a professional tool, and whilst they acknowledged there were no papers specific to radiography, they did contextualise their findings and discussion to radiography practice. However, they focused on SoMe's use as a professional development and teaching tool rather than as a means of engaging with patients. This was because their findings suggested engaging with patients was a high risk activity and one which gave practitioners most concern with regard to privacy, patient confidentiality and ethics.

In their 2016 study, Rosenkrantz et al [9] assessed 464 tweets from July to December 2015 using the hashtag mammogram. This study was conducted to evaluate themes in relation to patient experience of mammography and, though not related to radiography experience, did highlight an insight into the world of SoMe interaction within \#mammography. Within a powerful conclusion the authors highlighted the impact of women sharing experiences within a SoMe platform. They suggested further evaluation could warrant the use of SoMe within mammography as a tool for fostering adherence to guidelines and the sharing of experiences. 
There is therefore an overall lack of research about radiographers' attitudes towards the use of SoMe to engage with patients, yet a suggestion that anxieties may exist. In order to develop an online information hub which will bring patients and radiographers together we need to fully understand these anxieties and the difficulties these might pose for hub users. Having this information will assist us to ensure the relevant support and enablers are in place.

The aim of this study was therefore to explore breast screening practitioners' opinions and attitudes with regard to using SoMe to engage with clients online, identify challenges, and strategies to overcome them, and make recommendations for further work.

\section{Ethical Statement}

The study received ethical approval from the Higher Education Institution in which the WoMMeN research project is based. The study was conducted with due regard to participant confidentiality. All data was stored on password protected servers and no individuals have been named in the study. However, participants all gave consent to use of photographs of workshops for illustrative purposes and for this reason were aware that complete anonymity could not be guaranteed.

\section{Methodology}

The study used a qualitative research approach due to its exploratory nature.

Invitations were distributed to 82 Breast Screening Units (BSU) in England inviting participants to attend one of four workshops to be held in London, Manchester, Nottingham, and Leeds between December 2015 and March 2016. These sites were chosen as they were associated with the national academic and/or practice-based training sites. We were therefore able to work closely with the key informants (academic and clinical leads) to ensure effective dissemination of recruitment invitations and posters. The decision to hold four workshops was pragmatic and determined by financial constraints.

Those invited were:

- NHS Breast Screening Programme (NHS BSP) service managers - due to the nature of their role in allocating resources and time for mammographers to engage online with clients; 
- Training leads from the national training sites - as they would be responsible for driving the principles and practice of online communication into the curriculum of future trainees;

- A mammographer from each site - as the practitioners would be piloting recommendations emerging from the research.

News of the workshops spread within the breast screening community and a number of other personnel who had an interest in SoMe contacted us to ask if they may attend. As this was an exploratory workshop we did not reject any of these requests. Consequently there was representation from a range of roles including: administrators; public health practitioners, assistant practitioners; practitioners; unit managers; NHS BSP unit managers; educationalists; and those working at national strategic level within the NHS BSP (Fig 1). Therefore, for ease of reference, henceforth we refer to the participants as practitioners but it should be noted that this does not reflect their professional title.

The workshops included: a presentation about the use of SoMe in health; an informal 'audit' of the participants' use of SoMe (this was an ice-breaker rather than a data collection exercise); demonstration of good practice using Twitter (@weMammographers) and the WoMMeN hub, and a head-to-head video on one patient's perspective and use of SoMe to access breast screening-related information. We then collected the participants' views about using SoMe to engage with their own clients/service-users online about breast screening, exploring both challenges to using social media and solutions.

\section{Data Collection}

Nominal Group Technique (NGT) was utilised as the data collection tool. NGT allows the researcher to elicit views on a group basis, which exploits the advantage of constructing ideas in teams. However, individuals are then asked to rank the issues from their own perspective, thus eliminating the tendency for 'group-think' and to allow each individual to have a voice. Although the ranking system is a quantitative approach and does not sit easily within a qualitative study, it enables recommendations to be made about where to allocate limited resources [10].

Small groups of approximately 5 participants (mixed by sites and roles) were asked:

"What are the challenges that practitioners face in using SoME as part of their role?" 
Groups captured their thoughts on flip charts then each group's challenges were typed into an Excel spreadsheet that was projected on a screen for all groups to see. The whole group agreed the wording of the challenges and identified duplications so that challenges were not recorded more than once.

These challenges were then ranked for importance by participants acting as individuals. Due to time constraints, only the top four challenges, by rank, were reflected back to the group for identification of potential solutions. Each group of 5 was given one of the top four challenges to discuss and identify solutions. A second plenary session captured these thoughts which were again typed into the projected spreadsheet.

Throughout each workshop, flipchart memo notes were taken by a member of the team. These were visible for all participants in order to provide an element of participant verification.

\section{Results, Discussion and Recommendations}

Across the 4 workshops there were 78 participants from a wide geographical spread (Fig 2). Participants represented 50 of the 82 breast screening units to which invites were sent.

There were 19, 24, 20 and 28 challenges listed at each of the four workshops (Fig 3). The words and terms used to describe the challenges captured on the Excel spreadsheets were derived by the full cohort of participants at each workshop rather than being presented as predefined categories by the researchers. Because of this, wording of challenges at each workshop was slightly different. Therefore at the end of the four workshops the research team undertook a matching exercise to amalgamate identical challenges. We then themed the challenges into 5 categories; these fell into two broader overarching themes. The colour coding in figure 3 indicates which challenges were amalgamated into each theme.

The final themes were as follows:

\section{Theme 1: Working within boundaries}

- Professional/legal accountability

- Information accuracy (misinformation and misinterpretation)

- Time as a boundary

Theme 2: Support

- Employer:

- Access to technology 
- Skills and training

- Supportive policies

- Manager

The following discussion is framed around our interpretation and thematic coding of the challenges and supported by the memo notes taken during the workshop discussions. As the participants were tasked to come up with solutions to the challenges they had identified we present these after first discussing each challenge in more detail. In this way, the participants' solutions are articulated in the form of recommendations.

\section{Theme 1: Working within boundaries}

\section{Professional and legal accountability}

Participants were anxious about sharing personal information online, (their own and clients') with the overriding fear of inadvertently breaching patient confidentiality. Practitioners are accustomed to working within the structure of a large organisation whose culture is driven by notions of data protection and confidentiality, not the flattened hierarchy of the SoMe environment where 'sharing' abounds. Although as allied health professionals they should practise with autonomy this comes with a personal responsibility towards information governance.

Professional accountability is regulated by the Health and Care Professions Council (HCPC) and the Society and College of Radiographers (SCoR), and their Standards of Proficiency and Codes of Professional Conduct do not distinguish between faceto-face or online communication with regards to a health professional [11, 12]). If a mammographer is confident of their verbal communication skills then why treat the virtual environment any differently: don't say online what you would not say in a packed waiting room. The SCoR have produced SoME guidelines which echo this sentiment [13] and practitioners should feel heartened by this approbation.

However, it should also be noted that a number of the participants were not professionally qualified (i.e. registered with the AHP or other statutory body), for example office administrators and assistant practitioners. Participants pointed out that these people did not have the same obligations to act according to any specific professional code of conduct at all times. It was therefore less clear where they stood 
in terms of legal accountability and for these people clarity about employer position was even more important.

\section{Recommendations identified by participants:}

- It is important for breast screening practitioners to be aware of the relevant guidelines, e.g. from the SCoR and also the HCPC. Both encourage the use of SoMe and occasionally hold "tweetchats".

- There should be efforts to raise awareness of these guidelines.

- Breast screening personnel, who are not professionally registered, should make themselves aware of their own employer's policies (see 'Support' theme below)

\section{Information accuracy}

The results confirmed the findings of the literature: practitioners have clear anxieties about the use of SoMe when communicating about health, and in this instance, mammography and breast screening; yet this is a subject about which they hold specialist, experiential knowledge.

The study participants reported no fear in imparting knowledge face-to-face, but considerable anxiety about imparting accurate information and knowledge online, initially suggesting a lack of confidence in their professional knowledge. However, it was acknowledged that, as in the work environment, if an answer to a query wasn't known by the practitioner then it was acceptable to refer to another professional or reputable online source which did. This is accepted practice, as is the need for accuracy; both tenets are enshrined in the professional codes of conduct by the professional and regulating bodies [11, 12].

Participants were also anxious about being misinterpreted or inadvertently providing contextual information that would not be accurate for another breast screening centre.

\section{Recommendations identified by participants:}

- Practitioners require training in (i) how to use SoMe safely and (ii) how to communicate effectively using the written (online) word, e.g. addressing the 
lack of non-verbal cues. This training might be provided by websites, peers and in some cases IT departments

- Feedback on the value of the online response should be sought from clients.

- Provide 'information' only, not 'advice'

- In terms of accuracy, don't rush into answering queries, check information before posting, respond within and acknowledge scope of knowledge.

- An online community of practitioners would be useful for checking answers to difficult questions with peers and transferability to other sites.

- Links to websites should be checked to ensure that they are valid and reputable.

- Liaise with hospital's communications team to help check and phrase answers.

\section{Time as a boundary}

Participants expressed concern about the time required within the clinical environment to access SoMe. They were keen for dedicated time to be allocated on a rota for this work whereas managers tended to see engagement with clients online about breast screening information as part of a practitioner's core responsibility.

Traditional work-life boundaries are increasingly blurred, with the advent of ubiquitous smartphone use ensuring that we are connected both to work and social life constantly [4]. It has been demonstrated via one grounded theory study [14] that employees who used SoMe for personal use at work were more likely to use personal devices and time for work purposes, and that other forms of teleworking were not as effective if the employee did not blur boundaries in this manner. The research team advocated a flexible approach to SoMe use to improve team working across digital platforms, and also demonstrated that 'deterrence' policies were not effective.

\section{Recommendations identified by participants:}

Recommendations were less well defined for this challenge and very dependent on local working culture.

- To be determined locally:

- whether this should be done by one individual or all staff. 
- whether this should be allocated to a work rota or something a practitioner should do at any time - an aspect of their professional role

- A national initiative, rather than a site specific one would ensure a community of practitioners is more likely to be available at any time making more effective use of time.

\section{Theme 2: Support}

\section{Employer: Access, skills and training, supportive policies}

Another perceived challenge was access to technology, WiFi and SoMe platforms. The culture within the NHS, as reported by the participants, is generally to deny access: the apparent dichotomy between the NHS information strategy [5], which encourages online engagement with all stakeholders including the users of the service and the actual behaviour of individual hospitals, was confirmed by participants.

Participants also felt more could be done by employers in terms of providing skills training in the use of appropriate platforms and the nuances of effective online interaction. They felt a challenge to going online was knowing how to manage inappropriate posts and internet trolls and they would need training and support with this. They also felt policing of an online site would need to be in place to remove inappropriate posts.

More fundamentally, a tension still exists, with practitioners reporting that employers' policies created a barrier to an online presence. We followed this up by undertaking an audit of a selection of hospital communication policies in the North West of England. This confirmed the participants' view that some hospitals actively discourage staff from participating online, generally out of a need to protect the hospital's 'brand' and image [15].

\section{Recommendations identified by participants:}

- Practitioners should make themselves aware of their own employer's policies

- Where policies are unsupportive or discouraging of SoMe practitioners should take the initiative to lead change. 
- Cascade workshop presentations to communications teams and Senior Management Teams

\section{Manager}

Although some managers at the workshops were keen to explore the idea of SoMe use in their centres, a number of participants reported a lack of support from their managers. Indeed a small number of registrants had had to withdraw from the workshops at short notice claiming their managers had asked them not to attend. Overall, participants felt that without the support of their managers they would not be able to engage in communicating with clients in an online space.

Managers are clearly on the frontline when it comes to managing risk and need to constantly balance risk against benefit before introducing and/or supporting new initiatives such as SoMe. Participants suggested more evidence of benefit was needed to bring managers on board. To date such evidence is emergent but contradictory. Whilst two systematic reviews failed to find much high quality evidence that improved outcomes exist due to SoMe [16, 17]), a 2015 review by Smailhodzic et al [18] found improved communication between health professionals and patients as a result of its use. Nevertheless, no research exists to prove that SoMe improves outcomes or uptake in the field of mammography, and this is an area for further study.

\section{Recommendations identified by participants:}

- Generate evidence that the use of SoMe would have an effect on the numbers attending for breast screening.

- SoMe platforms for breast screening should be thoroughly evaluated using robust research methods.

- Promote the use of SoMe for breast screening through flyers and other promotional material which would build a critical mass of people online.

\section{Study Limitations}

Though the study had a wide reach with 78 attendees across a large geographical area, the overall geographic representativeness was limited. A representative from each BSU was sought though not every unit could be represented. Although it would be useful to elicit views from these units, this does not invalidate the usefulness of the findings. As Morse and Field 
note [19], in qualitative research, generalisability is not the aim. When reporting people's perceptions and experiences there is an acknowledgement that these may be infinite and context based. The aim is therefore not to capture all possible experiences but to capture as rich and truthful a picture of the participants' perspectives as possible. This ensures trustworthiness or credibility which we addressed in the study reported here through a number of measures: typing each group's own descriptions into the spreadsheets and projecting these for verification, and writing and displaying memos throughout the day, which again allowed participant verification. Furthermore, a team of four researchers themed the data which reduces the subjectivity associated with single researcher analysis. All participant comments were easily captured within the themes and there were no themes with less than 4 comments suggesting data saturation was approached.

\section{Conclusion}

Breast screening practitioners, as represented by our participants, appeared willing and motivated to engage in SoMe not only to benefit their clients but to improve their own professional networking and development. However, in-keeping with the literature from other disciplines, a number of anxieties existed. These related mainly to being unclear about whether they are supported by their employer and professional bodies. Our participants also identified a need to undergo training to develop their skills for communicating effectively online.

The problems of support and boundaries did not appear insurmountable and practitioners were able to propose realistic solutions. The proposed solutions would need to be driven not only by the enthusiastic early adopters but by professional bodies and employers.

\section{References}

1. Robinson L., Hogg P., Newton-Hughes A. (2013) The power and the pain: Mammographic compression research from the service-users' perspective Radiography 19 (3) 190-195

2. Robinson L., Griffiths M., Wray J., Ure C., Shires G., Stein-Hodgins J.R., Hill C., Hilton B. (2015) Preparing women for breast screening mammography: A feasibility study to determine the potential value of an online social network and information hub Radiography 21 (4) , pp. 308-314

3. Robinson L., Fenton A., Galpin A., Griffiths M., Hill C., Mercer C.. Meredith J., Scragg B., Shaikh S., Shires G., Stein Hodgins J., Ure C., Wray J., Taylor J. (2016) Word of Mouth Mammogram e-Network (WOMMeN) hub: a Social Media enabled 
client and practitioner collaboration (2016) Symposium Mammographicum, Liverpool UK

4. Ofcom (2016) Adults Media Use and Attitudes Report: section 5 Media Activities [available at] https://www.ofcom.org.uk/ data/assets/pdf file/0028/76177/section-5media-activities.pdf

5. Department of Health. (2012). The power of information: putting all of us in control of the health information we need. London: Department of Health. Retrieved from https://www.gov.uk/government/uploads/system/uploads/attachment data/file/21368 9/dh 134205.pdf

6. Lawson C., Cowling C. (2014) Social media: The next frontier for professional development in radiography (Review) Radiography 21 (2) 2014, Pages e74-e80

7. Moorhead S.A., Hazlett D.E., Harrison L., Carroll J.K., Irwin A., Hoving C.(2013) A New Dimension of Health Care: Systematic Review of the Uses, Benefits, and Limitations of Social Media for Health Communication. J Med Internet Res 15(4):e85 DOI: 10.2196/jmir.1933 PMID: 23615206 PMCID: $\underline{\text { PMC3636326 }}$

8. Grajales III F.J., Sheps S., Ho K., Novak-Lauscher H., Eysenbach G. (2014) Social Media: A Review and Tutorial of Applications in Medicine and Health Care. J Med Internet Res 16(2):e13 DOI: 10.2196/imir.2912 PMID: 24518354 PMCID: 3936280

9. Rosenkrantz, A.B., Labib, A., Pysarenko, K., Prabhu, V. (2016). What Do Patients Tweet About Their Mammography Experience? Academic Radiology. OnLine Sept 19 2016. DOI: http://dx.doi.org/10.1016/j.acra.2016.07.012

10. Gallagher M., Harres T., Spencer J., Bradshaw C., Webb I. (1993) The Nominal Group Technique: A Research Tool For General Practice? Family Practice 10 (1) pp 76-81

11. Health \& Care Professions Council. (2016). Standards of conduct, performance and ethics. London: Health \& Care Professions Council. Retrieved from http://www.hcpcuk.org/assets/documents/10004EDFStandardsofconduct,performanceandethics.pdf

12. Freeman C. (2013) Society and College of Radiographers. Code of Professional Conduct [available at] http://www.sor.org/learning/document-library/codeprofessional-conduct

13. Tucker K (2015) SoMeRAD: Guidance for the radiography workforce on the professional use of Social Media. London: Society and College of Radiographers. [available at] http://www.sor.org/learning/document-library/somerad-guidanceradiography-workforce-professional-use-social-media

14. Schalow P.R., Winkler T.J., Repschlaeger J., \& Zarnekow R. (2013). The Blurring Boundaries Of Work-Related and Personal Media Use: A Grounded Theory Study 
On The Employee's Perspective. In European Conference on Information Systems p.212.

15. Scragg B., Shaikh S., Robinson L. Mixed Messages an audit of NHS Trust Policies regarding use of Social Media (2016) Symposium Mammographicum, Liverpool UK. Poster presentation

16. Hamm M.P., Shulhan J., Williams G., Milne A., Scott S.D., \& Hartling L. (2014). A systematic review of the use and effectiveness of social media in child health. $B M C$ Pediatrics 14(1), 1-15. http://doi.org/10.1186/1471-2431-14-138

17. Rice M.S., Goodall J., Hetrick E.S., Parker G A., Gilbertson T., Amminger P.G., Alvarez-Jimenez M. (2014). Online and Social Networking Interventions for the Treatment of Depression in Young People: A Systematic Review. J Med Internet Res 16(9), e206. http://doi.org/10.2196/jmir.3304

18. Smailhodzic Edin Hooijsma, Wyanda Boonstra Albert and Langley David (2016) A systematic review of effects on patients and on their relationship with healthcare professionals J. Social media use in healthcare: BMC Health Services Research 16:442 [available at] https://bmchealthservres.biomedcentral.com/articles/10.1186/s12913-016-1691-0

19. Morse, J.M. Field, PA. (2002) Nursing Research: The Application of Qualitative Approaches. Nelson Thormes. Cheltenham 